\section{KOMPASS}

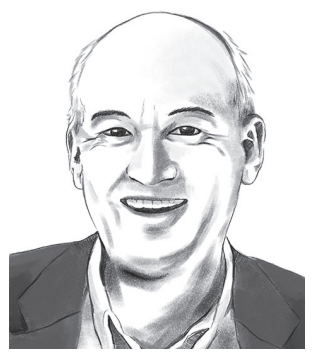

Harald Walach

Poznan/Berlin,

Polen/Deutschland

Wie würde sich das anhören, wenn wir bei einer Begrüßung sagen würden: «Na, du glykotoxischer Knuffel, wie geht's?» Das wäre eine akkurate Übersetzung von «Na, du süßer Knuffel» ins Wissenschaftsdeutsch. Seit Kurzem bahnt sich eine stille Revolution in der Ernährungslehre an. Der Trend eines halben Jahrhunderts, Fett für alle medizinischen Übel der Neuzeit verantwortlich zu machen, von koronarer Herzkrankheit bis zu metabolischem Syndrom und Adipositas, scheint gebrochen. Ein deutliches Zeichen war für mich, als JAMA INTERNAL MEDICINE die Arbeit von Historikern (!!!) veröffentlichte [1]. In den 1950er Jahren hatten nämlich Epidemiologen noch Zucker und Fett gleichermaßen als mögliche Ursachen für kardiovaskuläre Erkrankungen im Visier. Die historische Arbeit belegt, wie die Zuckerindustrie, mit Hilfe von ein paar strategischen Forschungsdollars dafür sorgte, dass ein Review die Waage in Richtung Fett zeigen ließ [2]. Fett, vor allem gesättigte Fettsäuren, waren von da ab der Schurke Nummer eins. Nationale Ernährungsleitlinien sorgten dafür, dass «Low-Fat» zu einem «Gesundheitsstandard» wurde. Fett wurde wo möglich

\title{
Unsere glykotoxische Ära geht zu Ende
}

\section{Harald Walach}

Poznań/Berlin, Polen/Deutschland

durch Kohlenhydrate - vor allem Einfachzucker in Bindemitteln, Fruchtzucker und dergleichen - ersetzt. Und die Leute wurden dicker und kränker, immerzu, sodass das metabolische Syndrom schon zur neuen Volksepidemie ausgerufen wurde.

Wir sind offenbar alle einem grandiosen Irrtum aufgesessen, international und global, ein halbes Jahrhundert lang.

Befeuert wurde das ganze durch Ancel Keys. Der publizierte selektierte Daten aus seiner «6-Länder-Studie» [3], die eigentlich eine «22-Länder-Studie» war. Die unpassenden Länder hatte er der Einfachheit halber weggelassen. So konnte er eine anscheinend perfekte Korrelation zwischen Konsum ungesättigter Fettsäuren und koronarer Herzkrankheit belegen. Dass die Daten von Keys gezinkt waren, haben übrigens Statistiker kurz darauf belegt [4], aber das wollte dann schon keiner mehr wissen. Wir sind offenbar alle einem grandiosen Irrtum aufgesessen, international und global, ein halbes Jahrhundert lang. Denn der Übeltäter ist nicht das Fett, sondern der
Zucker, genauer gesagt, die Kohlenhydrate der einfachen Sorte, die ja rasch in Zucker, und, wenn im Überfluss vorhanden, in Triglyceride umgebaut werden, und so in die Fettdepots wandern.

Das dürfte auch der Grund sein, warum ketogene Diäten, also solche, bei denen der Körper sich vor allem von Ketonkörpern ernährt, die bei der Verstoffwechslung von Fett erzeugt werden, allmählich beforscht werden $[5,6,7]$. Fast alle Körperzellen - außer Tumorzellen - können sich von solchen Ketonkörpern ernähren, auch unsere Neuronen. Deswegen fällt man auch beim Fasten nicht ohnmächtig um. Und einige Forscher gehen sogar davon aus, dass Alzheimer und Demenzerkrankungen als Typ-III-Diabetes angesprochen werden sollten: Aufgrund der Insulinresistenz der Neuronen verhungert das Gehirn trotz - oder aufgrund - eines Überangebots von Zucker [8].

Vielleicht sagen wir jetzt in Zukunft: «Na, du fetter Knuffel»?

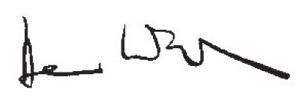

Prof. Dr. Dr. phil. Harald Walach

\section{Literatur}

-1 Kearns CE, Schmidt LA, Glantz SA: Sugar industry and coronary heart disease research: A historical analysis of internal industry documents. JAMA Intern Med 2016;176: 1680-1685.

2 McGandy RB, Hegsted DM, Stare FJ: Dietary fats, carbohydrates and atherosclerotic vascular disease. N Engl J Med 1967;277:186-192.

13 Keys A: Atherosclerosis: A problem in newer public health. J Mt Sinai Hosp N Y 1953;20: 118-139.

4 Yerushalmy J, Hillboe HE: Fat in the diet and mortality from heart disease. A methodologic note. N Y State J Med 1957;57:2343-2354.

$\checkmark 5$ Abbasi J: Interest in the ketogenic diet grows for weight loss and type 2 diabetes. JAMA 2018;319:215-217.

6 Bueno NB, de Melo ISV, de Oliveira SL, da Rocha Ataide T: Very-low-carbohydrate ketogenic diet $\mathrm{v}$. low-fat diet for long-term weight loss: a meta-analysis of randomised controlled trials. Br J Nutr 2013;110:1178-1187.
Klement RJ: Fasting, Fats, and Physics: Combining Ketogenic and Radiation Therapy against Cancer. Complement Med Res 2018; 25:102-113.

$>8$ de la Monte S, Wands JR: Alzheimer's Disease is type 3 diabetes - evidence reviewed. J Diabetes Sci Technol 2008;2:1101-1113.

\section{KARGER}

๑๑) 2019 S. Karger GmbH, Freiburg

Fax +497614520714

information@karger.com

www.karger.com 